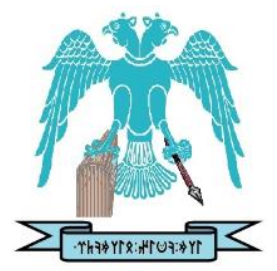

JOURNAL OF ENERGY SYSTEMS

$2021,5(3)$

\title{
Sensorless current prediction in single phase inverter circuits with machine learning algorithms
}

\author{
Huseyin Ture \\ Karamanoglu Mehmetbey University, Graduate School of Natural and Applied Sciences, Department of \\ Electrical and Electronics Engineering, Karaman, Turkey, huseyinture00@gmail.com \\ Selami Balci* \\ Karamanoglu Mehmetbey University, Faculty of Engineering, Department of Electrical and Electronics \\ Engineering, Karaman, Turkey, sbalci@kmu.edu.tr \\ Muhammet Fatih Aslan \\ Karamanoglu Mehmetbey University, Faculty of Engineering, Department of Electrical and Electronics \\ Engineering, Karaman, Turkey, mfatihaslan@kmu.edu.tr
}

Kadir Sabanci

Karamanoglu Mehmetbey University, Faculty of Engineering, Department of Electrical and Electronics Engineering, Karaman, Turkey, kadirsabanci@kmu.edu.tr

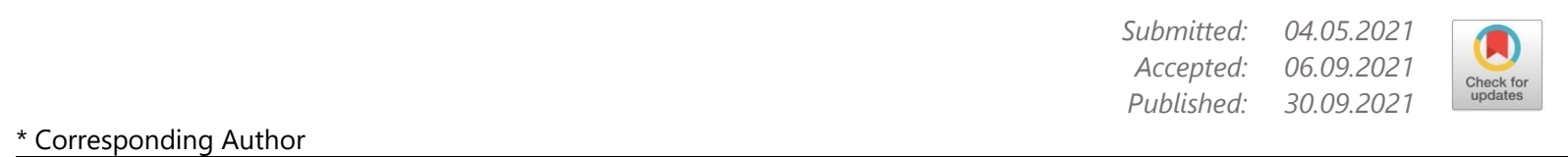

Abstract: Inverter circuits are widely used in power electronics applications such as electric motor control, induction heating or different Alternating Current (AC) loads. The control signal applied to the switching elements can affect the quality of the sinusoidal signal that occurs at the output of the inverter circuit by the means of voltage and current values. The inverter circuit topologies are generally designed as closed loop. However, these cause complexity of the circuit topology, increase the production costs and give difficulties in designing the control signals. In the present work, simulations of a single-phase inverter circuit with the Sinusoidal Pulse Width Modulation (SPWM) control signal are performed. Thus, the effect of the SPWM signals on the output of the inverter has been observed by changing the Modulation rate $(\mathrm{M})$, the carrier signal frequency $\left(f_{c}\right)$ and the reference signal frequency $\left(f_{r}\right)$. Through the data obtained, the output current of a phase inverter without a sensor is estimated by Machine Learning Algorithms (MLA) such as MultiLayer Perceptron (MLP), Support Vector Machine (SVM) and K-Nearest Neighbors (KNN).

Keywords: Inverter circuit, Machine learning algorithms, Sensorless control, Parameter estimating

$\begin{array}{cc} & \text { Ture, H., Balci, S., Aslan, M.F., Sabanci, K Sensorless current prediction in single phase } \\ \text { Cite this paper as: } & \text { inverter circuits with machine learning algorithms. Journal of Energy Systems 2021; } \\ & \text { 5(3), 221-230, DOI: } 10.30521 / \text { jes. } 932581\end{array}$

(c) 2021 Published by peer-reviewed open access scientific journal, JES at DergiPark (https://dergipark.org.tr/en/pub/jes) 


\section{INTRODUCTION}

Generally, from direct current (DC) to alternative current (AC) power converter circuits are known as inverters, and they can be designed in one phase, three phase or multi-phase according to the output to operate AC loads. Thanks to the inverters, it is possible to adjust the output voltage and frequency, thereby, inverters, which are frequently used in industrial applications, are widely used in the control of various motor types and in power systems. While the outputs of ideal inverters are sine waves, this is not possible due to the various losses we encounter in practice. The output is tried to get closer to the sinus wave with different gate signals and inverter topologies. The higher the level of inverter topology used, the more similar to the output sine. However, the gate control of switching elements such as MOSFET, IGBT, etc., becomes difficult and the cost increases [1,2].

The basic Pulse Width Modulation (PWM) used to control the gate signals of the switching elements of the inverters is the most used gate control signal in the industry as it is simple to design and implement. However, due to its inefficiency, different PWM techniques have been developed in recent years. Low total harmonic distortion (THD), low current ripple and variable output voltage have made the sinusoidal PWM method more common than traditional PWM [3, 4]. The signal obtained by comparing a sine wave and a triangle wave, Sinusoidal Pulse Width Modulation (SPWM), provides distortion factor and lower harmonic distortion for the inverter circuits. This technique used in inverters is frequently finds its place in the industrial applications due to its easy design, the output voltage resembling pure sinusoidal and its reliability $[5,6]$.

The SPWM technique is preferred in applications producing different voltage levels, for example at variable wind speeds such as wind energy systems, because of its easy applicability, low cost and low Total Harmonic Distortion (THD) [7]. For this reason, it is used as the control signal of the circuit topologies used to stabilize the network voltage [8]. The SPWM is used as a control signal in Uninterruptible Power Supplies (UPS) to provide emergency power to critical loads in case of main failure [9]. Solanki et al., [10] performed a performance comparison between PWM and SPWM of a three-phase induction motor. On the other hand, Maswood [11] investigated the switching loss of IGBT used as a power switch in a three-phase inverter with the SPWM gate control signal. Various physical sensors are used to detect the output current of the inverter. However, these external sensors cause complexity of the circuit. Today, there are machine learning algorithms developed for such closed loop systems. This is because machine learning algorithms offer much more practical and precise solutions in mathematical calculations or applications that are difficult to try and measure [12].

In this study, the parametric simulation has been performed for single phase inverter using SPWM control technique in Ansys - Simplorer software, and the output current of the inverter is estimated by Machine Learning Algorithms (MLA) without using a sensor. In the second section, information about simulation study and inverter is given. In the third section, machine learning and prediction results are given. The contributions of the method proposed in this study are listed as follows:

- The parametric simulation studies using SPWM control for the inverter circuits.

- Estimating the output current of the inverter using machine learning algorithms without using sensors.

- Determining the best parameter values for different machine learning algorithms. 


\section{PWM AND SPWM INVERTER CIRCUITS}

Inverters using PWM switching technique transform the signal from a constant DC source into an AC signal whose voltage and frequency can be controlled [13]. By adjusting the PWM technique duty cycle, the voltage and PWM frequency can be changed and the output frequency can be controlled. Fig. 1 is the gate trigger signal, and Fig. 2 shows the square wave and sinusoidal signal generated at the inverter output with a PWM signal.

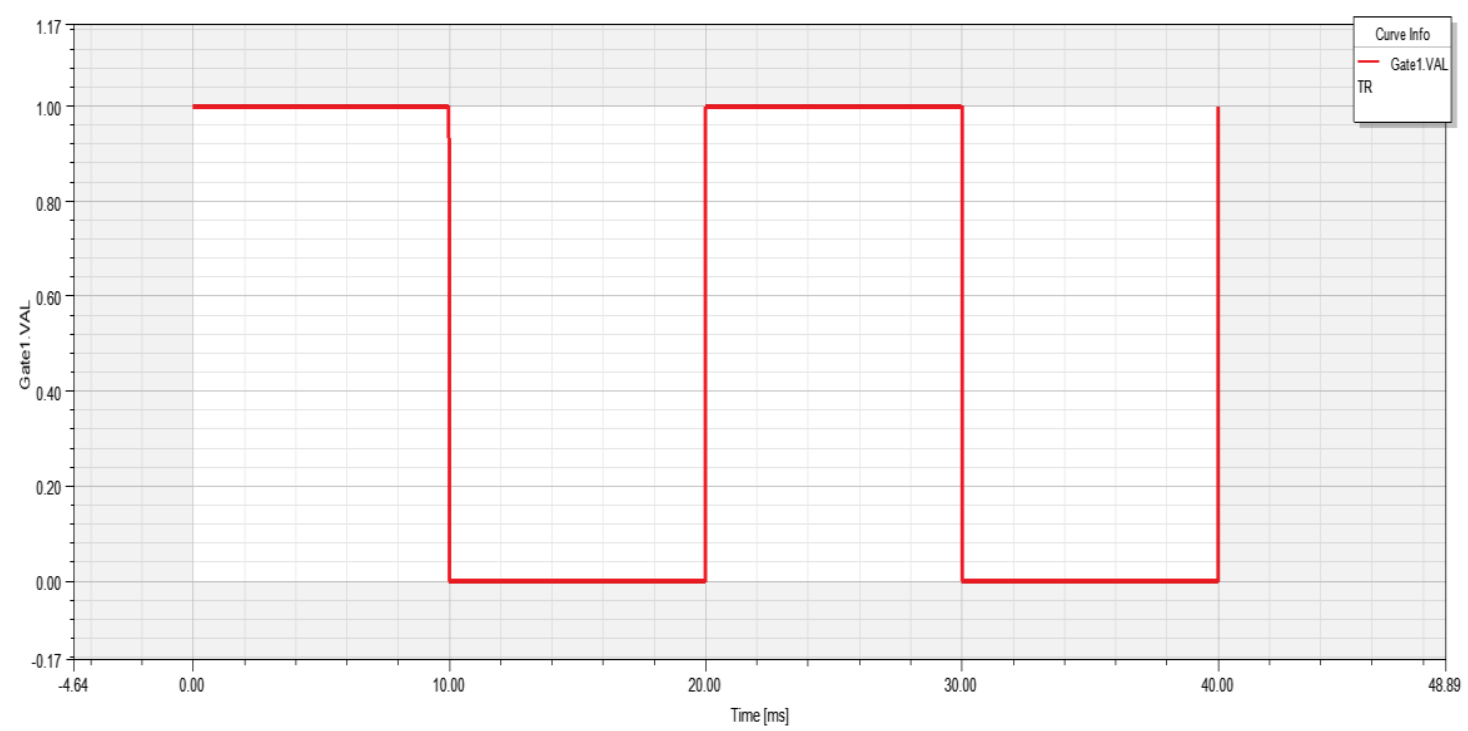

Figure 1. Switching gate signal in inverter circuit

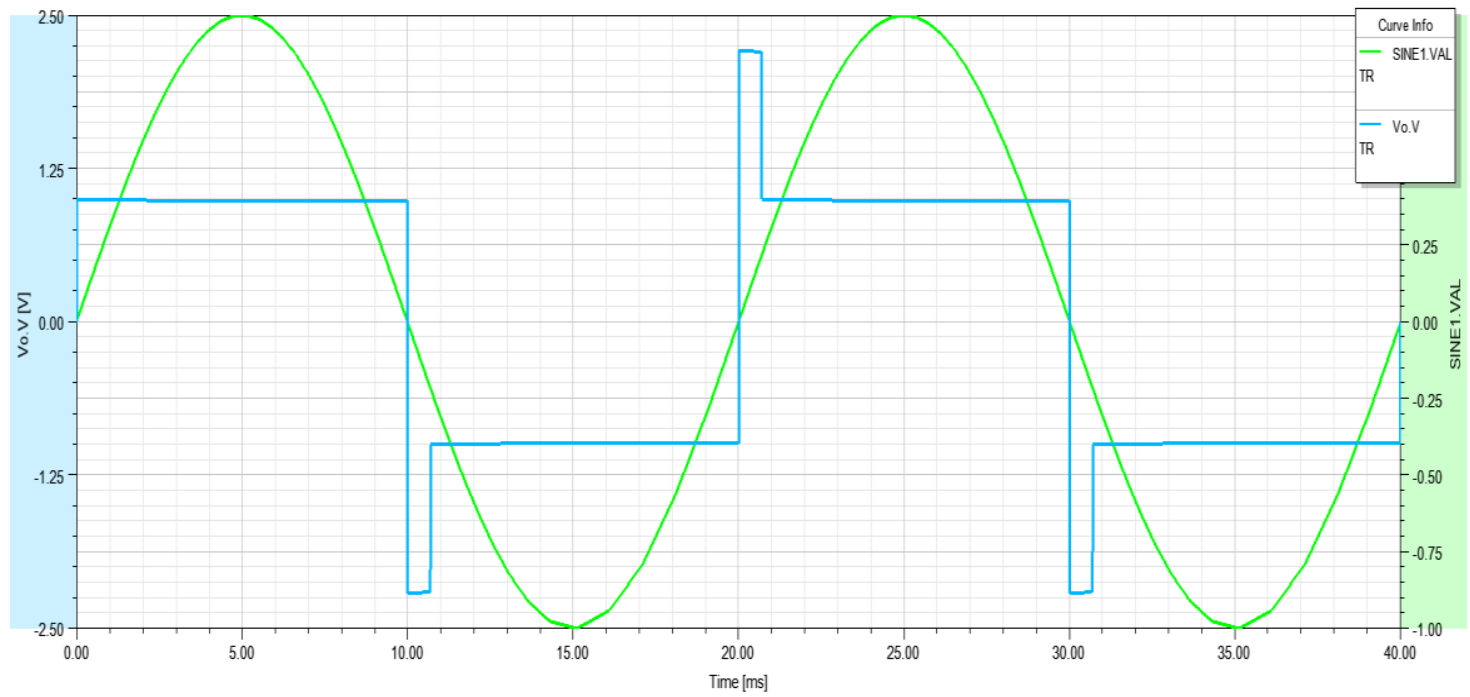

Figure 2. The square wave and sinusoidal signal generated at the inverter output with a PWM signal.

In this study, SPWM, is used as a gate control signal in inverters. This signal is obtained by comparing the carrier triangle signal with the reference sine signal. The signal resulting from the comparison is used for opening and closing power switches in inverters or power electronics [14]. Output voltage $V_{o}$ can be calculated as shown in Eq. 1 .

$$
V o=M V d=\frac{V c}{V t r} V d
$$


The ratio of the amplitudes of the carrier signal and the reference signals gives the modulation ratio $(M)$. The output voltage is adjusted with the modulation ratio. Thus, the SPWM control signal is shown in Fig. 3.

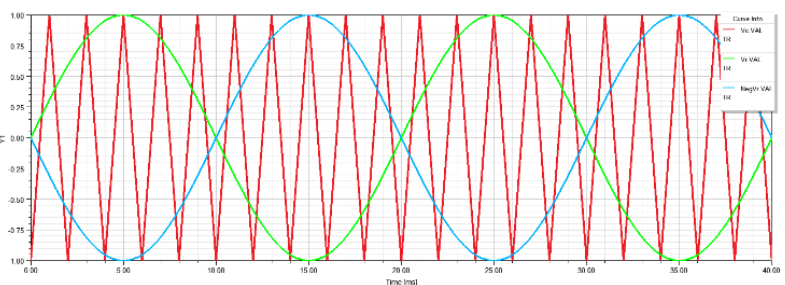

(a) Comparison of triangular signal and sinusoidal signal

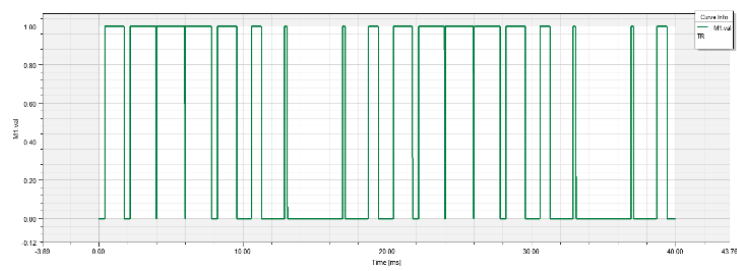

(b) SPWM gate trigger signal

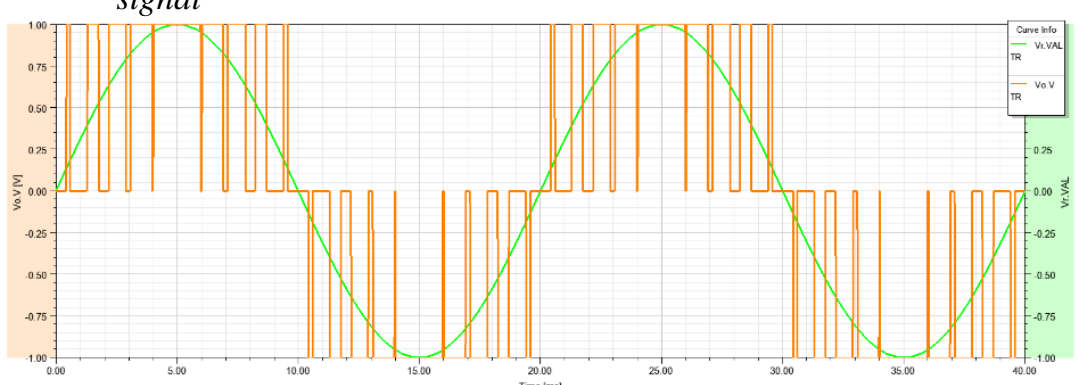

(c) Comparison of inverter output voltage and sinusoidal signals

Figure 3. The SPWM switching control signal

The number of pulses $(\mathrm{Pr})$ shown in Eq. 2 causes the ripple rate at the inverter output to change.

$$
\operatorname{Pr}=\frac{f c}{f r}
$$

In Fig. 4, the current ripple of the carrier signal at different frequencies is shown by keeping the reference signal constant at $100 \mathrm{~Hz}$ and the modulation ratio at 0.8 .

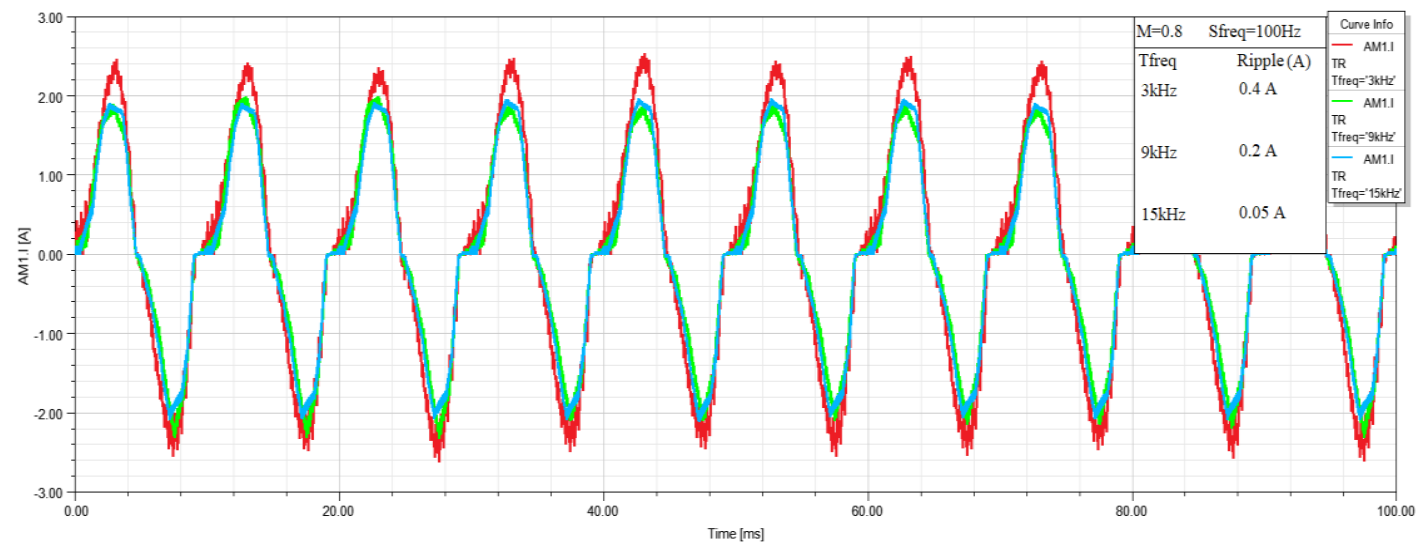

Figure 4. Inverter current graph generated by the SPWM trigger signal and ripple values.

\section{SIMULATION STUDIES}

If circuits modeled with power electronics circuit software can be simulated more realistically, results very close to experimental studies can be obtained. In this case, one or more of the circuit parameters 
can be changed to develop a realistic model for the parametric simulation studies [12]. Thus, using the dataset obtained as a result of the parametric analysis, realistic values are obtained with machine learning algorithms. In this study, single phase inverter circuit is modeled as shown in Fig. 5.

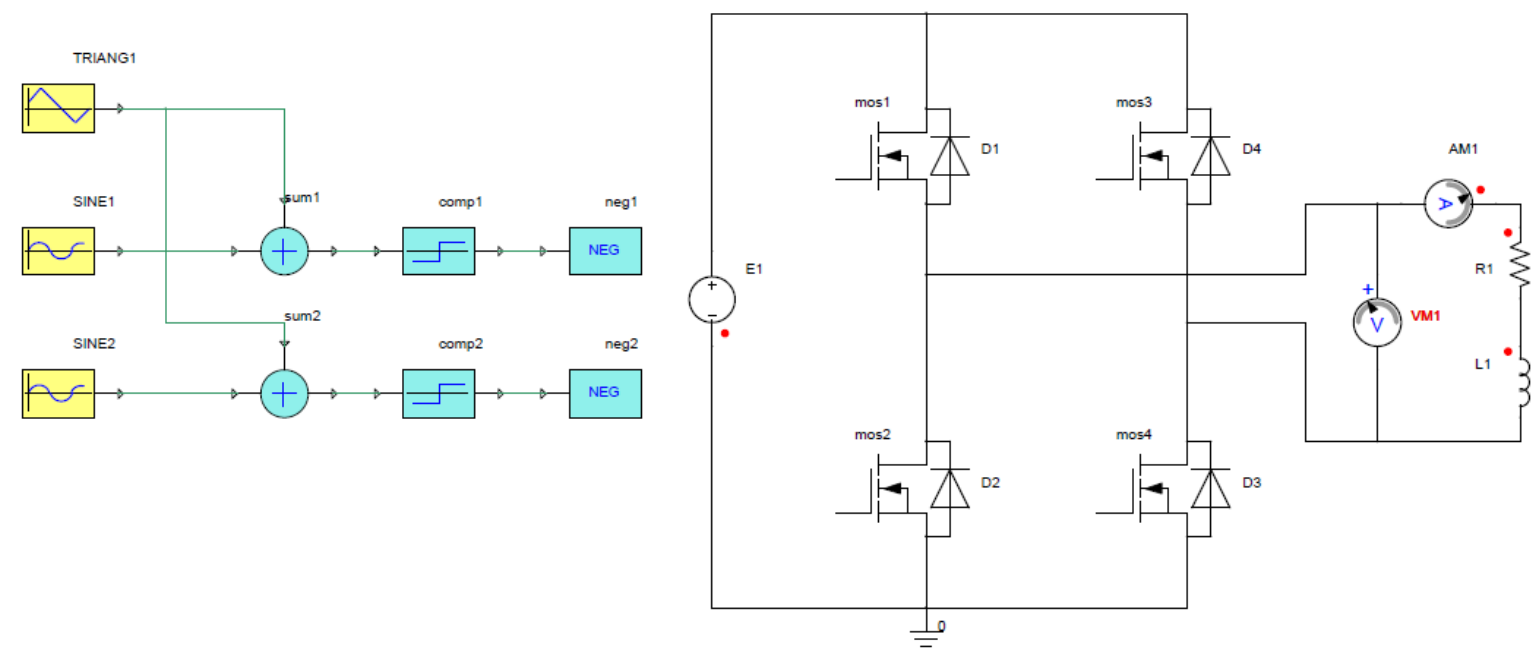

Figure 5. Parametric analysis circuit with ANSYS-Simplorer.

In the simulation study, the parametric analysis is performed for the single-phase inverter with different SPWM control signals as shown in Table 1 . In this context, the modulation ratio $(M)$, which will affect the output of the inverter, has been changed between 0.5-1 with 0.1 steps. In order to see the pulse number change, the carrier signal frequency $\left(f_{c}\right)$ is changed in $1.5 \mathrm{kHz}$ steps between $3 \mathrm{kHz}-15 \mathrm{kHz}$, and the reference signal frequency $\left(f_{r}\right)$ is changed in $100 \mathrm{~Hz}$ steps between $100 \mathrm{~Hz}-500 \mathrm{~Hz}$. In total, 486 different results are obtained. Therefore, Fig. 6 shows inverter output current graphs, and, Fig. 7 shows the inverter output current rms value.

Table 1. Technical characteristics of the parametric simulation.

\begin{tabular}{ccc}
\hline Parameters & Value & Number of steps \\
\hline$V_{i}$ & $12 \mathrm{~V}$ & constant \\
$M$ & $0.5-1$ & 0.1 \\
$f_{c}$ & $3-15 \mathrm{kHz}$ & $1.5 \mathrm{kHz}$ \\
$f_{r}$ & $100-500 \mathrm{~Hz}$ & $100 \mathrm{~Hz}$ \\
$R_{L}$ & $3-9 \mathrm{ohms}$ & $1.76 \mathrm{ohms}$ \\
\hline
\end{tabular}

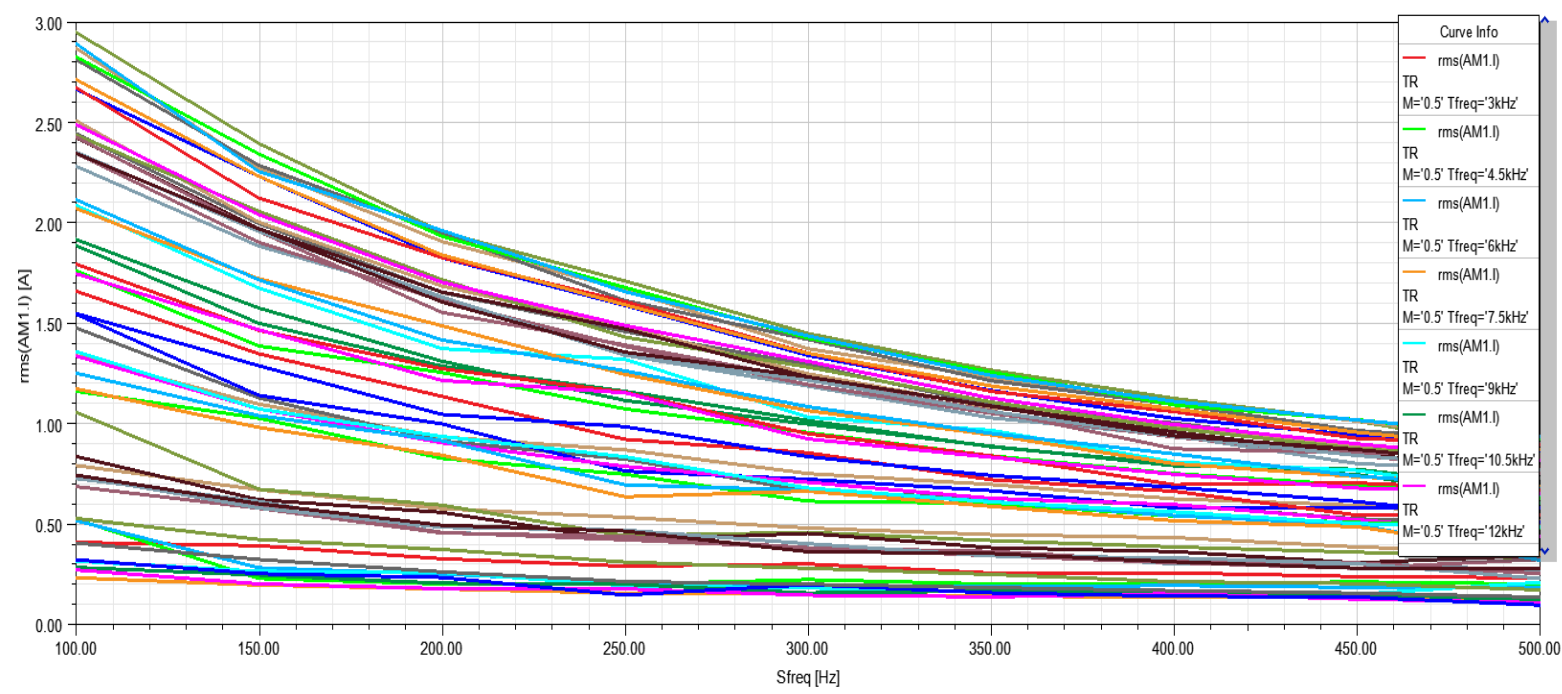

Figure 6. Output current rms values of the inverter circuits 


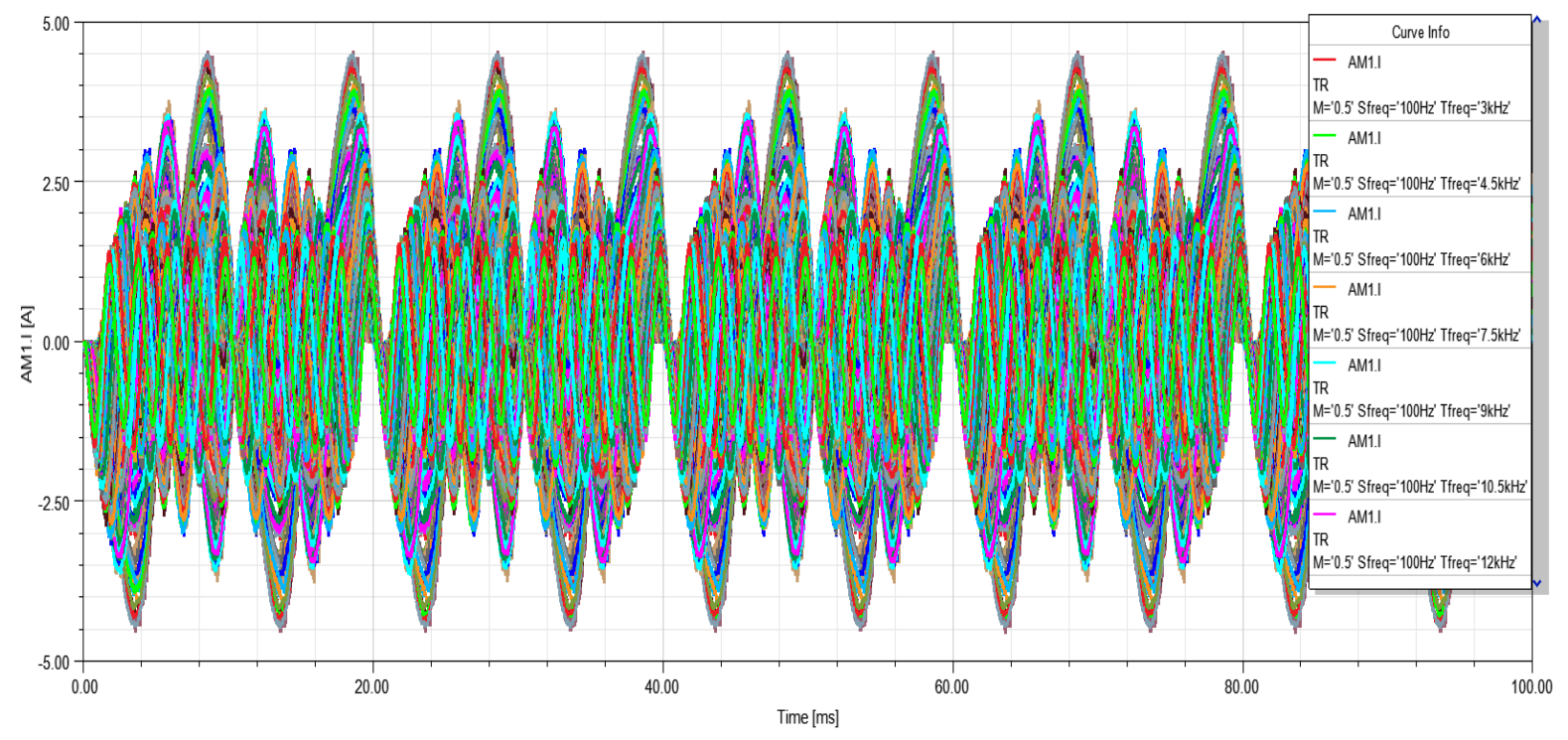

Figure 7. The parametric waveforms of the inverter output current.

\section{MACHINE LEARNING ALGORITHMS}

\subsection{Multilayer Perceptron}

Multi-Layer Perceptron (MLP) is a feed forward Artificial Neural Network (ANN) class consisting of at least one input layer and one output layer [15]. ANN is designed based on statistical learning theory, inspired by biological neurons to process information. The classification, estimation and pattern recognition are performed with ANN. Artificial neurons are composed of interconnecting weights, and as these weights are updated, the output value approaches the target value. The update process is repeated until the statistically optimal value is obtained. As a result, the ANN model is trained and test data is used to measure the performance of its network [16-19].

\subsection{K-Nearest Neighbor}

K-Nearest Neighbor (KNN) algorithm is a basic method that is easy to learn and understand and is considered the simplest method of machine learning. KNN finds the $k$ closest neighbors of a point in the feature space and then estimates the point class based on $k$ distances from the nearest neighbors [2022].

\subsection{Support Vector Machine}

Support Vector Machine (SVM) is developed based on statistical learning theory. SVM maps the dataset from the input space to a higher dimensional feature space, thus the classification problem becomes easier [23]. In the feature space, a boundary plane (hyperplane) is drawn between the classes. Two parallel hyperplanes are formed on both sides of the plane separating the data, and the distance between these planes affects the performance of the classifier. In cases where the data are not linear, a different high property space is created, and in this way the data can be classified linearly [24, 25]. 


\section{RESULTS}

Machine Learning based MLP, SVM and KNN applications were applied to estimate the output current. Since these methods contain many different parameters in their structure, MLP, SVM and KNN models that provide the best estimation have been investigated. For this purpose, different models are developed using different parameters. The general architecture of the study is shown as block diagram in Fig.v8.

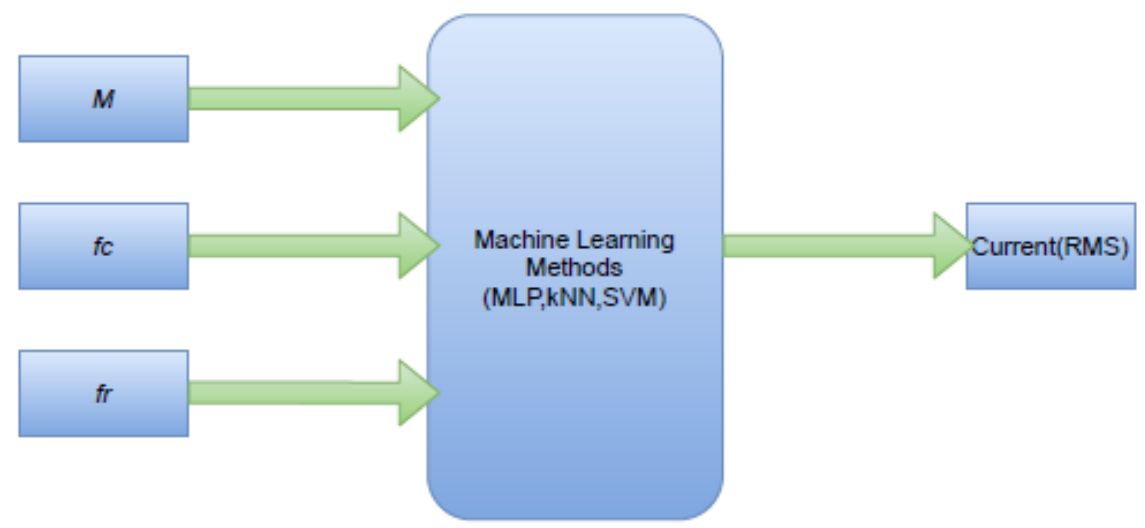

Figure 8. General block diagram of the application.

The best performance parameter values of machine learning algorithms were found by using trial and error method. The best performance parameter results are shown in Table 2. 486 data are obtained as a result of the simulation. During the training of machine learning algorithms, $80 \%$ of the data set (389 data) are randomly selected. The remaining $20 \%$ (97) data were used to evaluate the performance of the trained model. Root Mean Square Error (RMSE) and Mean Absolute Error (MAE) criteria are used as performance measurement criteria. Equations for these criteria are given in Equation 3 and Equation 4. The metric value results, correlation coefficient and learning time calculated for each machine learning method are given in Table 3 .

Table 2. Best parameter values for different machine learning algorithms.

\begin{tabular}{l|l|l}
\hline Model & \multicolumn{1}{|c}{ Parameters } & \multicolumn{1}{c}{ Type / Value } \\
\hline \multirow{4}{*}{ MLP } & Epochs & 500 \\
& Learning Rate & 0.3 \\
& Momentum & 0.2 \\
& Learning algorithm & Levenberg-Marquardt \\
& Activation function & Sigmoid \\
& Hidden layer & 1 \\
& Number of hidden layer neurons & 6 \\
\hline \multirow{2}{*}{ KNN } & Distance Function & Euclidean Distance \\
& $k$ neighborhood value & 2 \\
\hline \multirow{2}{*}{ SVM } & Kernel function & Pearson VII kernel function (PUK) \\
& Complexity constant (C) & 1.8 \\
& Omega & 2.75 \\
& Sigma & 2.0 \\
\hline
\end{tabular}

$$
\mathrm{RMSE}=\sqrt{\sum_{i=1}^{N} \frac{(\text { output }- \text { target })^{2}}{N}}
$$




$$
M A E=\frac{1}{N} \sum_{i=1}^{N} \mid \text { output }- \text { target } \mid
$$

Table 3. Error values according to the best parameters.

\begin{tabular}{lllll}
\hline MLA & MAE & RMSE & Correlation coefficient & Learning time $(\mathrm{sec})$ \\
\hline MLP & 0.0567 & 0.0833 & 0.9915 & 0.17 \\
KNN & 0.0650 & 0.1048 & 0.9848 & 0.01 \\
SVM & 0.0501 & 0.0812 & 0.9907 & 0.12 \\
\hline
\end{tabular}

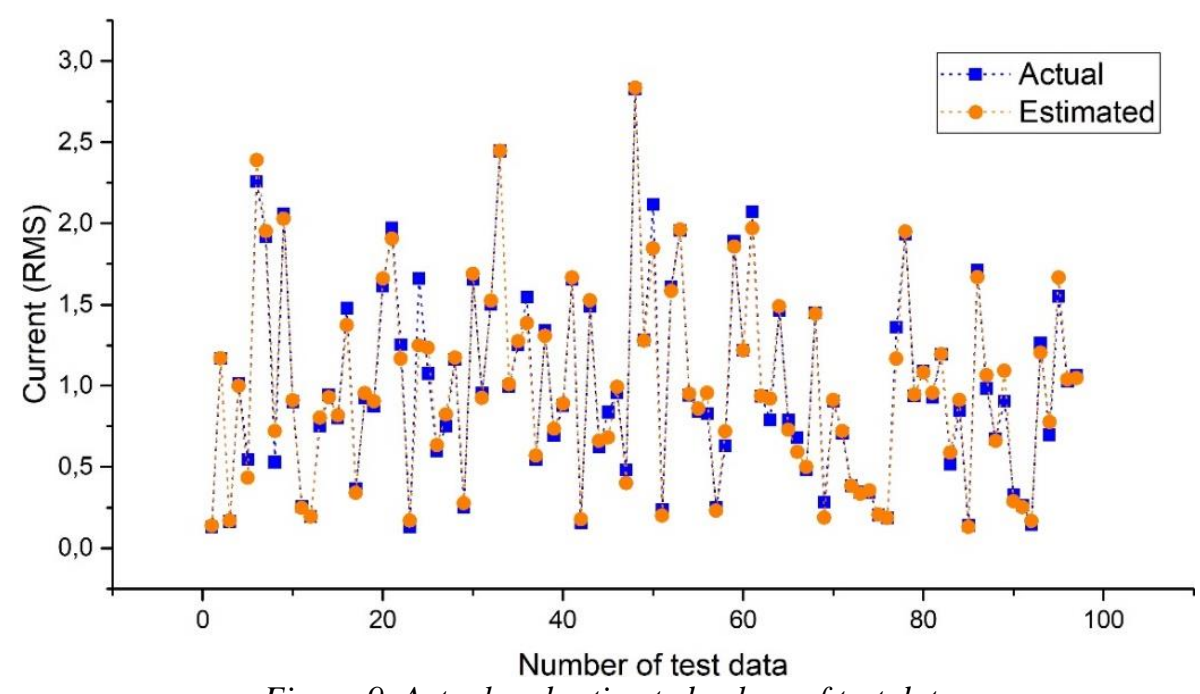

Figure 9. Actual and estimated values of test data

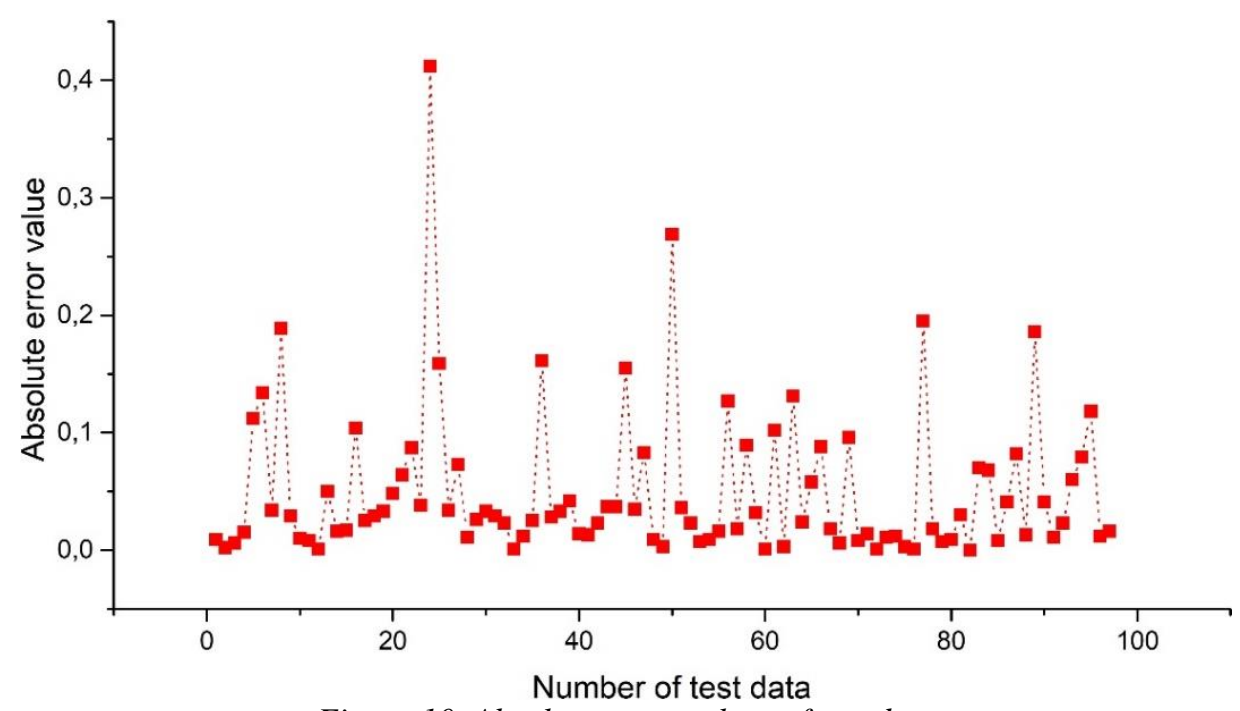

Figure 10. Absolute error values of test data 
When the values in Table 3 are examined, it is seen that all MLT make a very good estimate. However, the best results are obtained with SVM. The estimated values and actual values obtained with SVM are shown in Fig. 9. The absolute error values of the test data are shown in Fig. 10.

\section{CONCLUSION}

In this study, the parametric simulation studies have been done for single-phase inverter circuit. The data sets obtained from the parametric simulation result were divided into two groups as $80 \%$ and $20 \%$. While $80 \%$ of the data set was used for training, the remaining $20 \%$ was used to test the model performance. Machine learning algorithms MLP, KNN and SVM are used for current estimation of single-phase inverter. In order to obtain the best results, the parameters of the algorithms are determined by changing them manually. MAE and RMSE metrics were used to evaluate the performance of different machine learning algorithms. The results show that the output current of the inverter can be predicted highly accurately by machine learning algorithms. Especially with the SVM method, more successful results were obtained compared to other methods. In future studies, studies on controlling the current value of various motors (DC and AC) without using sensors will be carried out.

\section{REFERENCES}

[1] Rahim, NA., Chaniago, K., Selvaraj, J. Single-phase seven-level grid-connected inverter for photovoltaic system, IEEE transactions on industrial electronics 2010; 58: 2435-2443.

[2] Rashid, MH. Power Electronics-Circuits, Devices and Applications. Burlington, USA: ButterworthHeinemann, 2010.

[3] Bhattacharjee, T, Jamil,M, Jana, A. Design of SPWM based three phase inverter model. In: ICSESP 2018 Technologies for Smart-City Energy Security and Power; 28-30 March 2018: IEEE, pp. 1-6, doi: 10.1109/ICSESP.2018.8376696.

[4] Ismail, B, Taib, S, Saad, ARM, Isa, M, Hadzer, CM. Development of a Single Phase SPWM MicrocontrollerBased Inverter. In: PECon 2006 IEEE International Power and Energy Conference; 28-29 November 2006: IEEE, pp. 437-440.

[5] Liou, WR, Villaruza, HM, Yeh, ML, Roblin, P. A digitally controlled low-EMI SPWM generation method for inverter applications. IEEE Transactions on industrial informatics 2013; 10: 73-83.

[6] Maheshri, S, Khampariya, P. Simulation of single phase SPWM (Unipolar) inverter. International journal of innovative research in advanced Engineering 2014; 1(9):12-18.

[7] Karthikeyan, B, Sundararaju, K, Palanisamy, R. ANN-Based MPPT Controller for PEM Fuel Cell Energized Interleaved Resonant PWM High Step Up DC-DC Converter with SVPWM Inverter Fed Induction Motor Drive. Iranian Journal of Science Technology, Transactions of Electrical Engineering 2021; 1-17.

[8] Xue, Y, Chang, L. Closed-loop SPWM control for grid-connected buck-boost inverters. In: IEEE 2004 35th Annual Power Electronics Specialists Conference; 20-25 June 2004: IEEE, pp. 3366-3371.

[9] Zhang, K, Kang, Y, Xiong, J, Chen, J. Direct repetitive control of SPWM inverter for UPS purpose. IEEE Transactions on Power Electronics 2003; 18: 784-792.

[10] Solanki, G., Gurjar, C., Lokhande, M. Modelling and Performance Evaluation of Square Wave And Spwm Based Inverter Fed AC Drive. International Journal for Research in applied Science EngineeringTechnology 2014; 2: 244-248.

[11] Maswood, AI. A switching loss study in SPWM IGBT inverter. In: PECon 2008 2nd International Power and Energy Conference;1-3 December 2008: IEEE, pp. 609-613.

[12] Sabanci, K, Balci, S, Aslan, MF. Estimation of the switching losses in DC-DC boost converters by various machine learning methods. Journal of Energy Systems 2020; 4: 1-11.

[13] Raju, NI, Islam, MS, Uddin, AA. Sinusoidal PWM signal generation technique for three phase voltage source inverter with analog circuit \& simulation of PWM inverter for standalone load \& micro-grid system. International journal of renewable energy research 2013; 3: 647-658.

[14] Lakka, M, Koutroulis, E, Dollas, A. Development of an FPGA-based SPWM generator for high switching frequency DC/AC inverters. IEEE Transactions on power electronics 2013; 29: 356-365. 
[15] Aslan, MF, Sabanci, K, Durdu, A. Different wheat species classifier application of ANN and ELM. Journal of Multidisciplinary Engineering Science Technology 2017; 4: 8194-8198.

[16] Darvishan, A., Bakhshi, H., Madadkhani, M., Mir, M., Bemani, A. Application of MLP-ANN as a novel predictive method for prediction of the higher heating value of biomass in terms of ultimate analysis. Energy Sources, Part A: Recovery, Utilization, Environmental Effects 2018; 40: 2960-2966.

[17] Gupta, DK. A review on wireless sensor networks. Network Complex Systems 2013; 3: 18-23.

[18] Kayabasi, A. An Application of ANN Trained by ABC Algorithm for Classification of Wheat Grains. International Journal of Intelligent Systems Applications in Engineering 2018; 6: 85-91.

[19] Sabanci, K, Kayabasi, A, Toktas, A. Computer vision-based method for classification of wheat grains using artificial neural network. Journal of the Science of Food Agriculture 2017; 97: 2588-2593.

[20] Koklu, M, Ozkan, IA. Multiclass classification of dry beans using computer vision and machine learning techniques. Computers Electronics in Agriculture 2020; 174: 105507.

[21] Sabanci, K, Koklu, M. The Classification of Eye State by Using kNN and MLP Classification Models According to the EEG Signals. International Journal of Intelligent Systems Applications in Engineering 2015; 3: 127-130.

[22] Zhang, S, Cheng, D, Deng, Z, Zong, M, Deng, X. A novel kNN algorithm with data-driven k parameter computation. Pattern Recognition Letters 2018; 109: 44-54.

[23] Deka, PC. Support vector machine applications in the field of hydrology: a review. Applied soft computing 2014; 19: 372-386.

[24] Durgesh, KS, Lekha, B. Data classification using support vector machine. Journal of theoretical applied information technology 2010; 12: 1-7.

[25] Sabanci, K. Artificial intelligence based power consumption estimation of two-phase brushless DC motor according to FEA parametric simulation. Measurement 2020; 155: 107553. 